\title{
Effects of ivabradine on ventricular electrophysiological remodeling after myocardial infarction in rats
}

Li-ping Jiang ${ }^{1}$, Jing-jing Lin², Ting-pei Zhuang², Wei-wei Wang², Hang-zhou Wü ${ }^{3}$, Fei-long Zhang²

\author{
${ }^{1}$ Department of General Medicine, Mengchao Hepatobiliary Hospital of Fujian Medical \\ University, Fuzhou, China \\ 2Department of Cardiology, Fujian Medical University Union Hospital and Fujian \\ Provincial Institute of Coronary Disease, Fuzhou, China \\ ${ }^{3}$ Medical Insurance Office, Fujian Medical University Union Hospital, Fuzhou, China
}

Submitted: 10 July 2020

Accepted: 22 October 2020

Arch Med Sci

DOI: https://doi.org/10.5114/aoms.2020.101181

Copyright $\odot 2020$ Termedia \& Banach

\section{A bstract}

Introduction: This study aims to investigate the effects of ivabradine (IVA) on ventricular electrophysiological remodeling after myocardial infarction (MI) in rats.

Material and methods: A total of 60 male Sprague-Dawley rats were randomly divided into five groups: an MI group, an IVA group, a metoprolol (MET) group, an IVA + MET group, and a sham group. After a four-week intervention, the ventricular electrophysiological parameters were detected by multichannel electrophysiological polygraph. Then, the morphological characteristics were evaluated using hematoxylin and eosin ( $\mathrm{H} \& \mathrm{E})$ and Masson's staining, and the expression of phosphorylated connexin 43 (p-Cx43) in the left ventricular wall was detected through immunohistochemistry and the Western blot test.

Results: The electrophysiological examination revealed that the induction rate and fatality rate of ventricular tachycardia (VT)/ventricular fibrillation (VF) were lower in both the IVA and the MET group, compared with the MI group $(6 / 12,6 / 12$ vs. $10 / 11$; and $1 / 12,1 / 12$ vs. $5 / 11$; all $p<0.05)$, as well as the IVA + MET group (1/11 vs. $10 / 11, p<0.01$; and $1 / 11$ vs. $5 / 11, p<0.05)$ The induction rate of VT/VF was lower in the IVA + MET group, compared to the MET group ( $1 / 11$ vs. $6 / 12, p<0.05)$. H\&E and Masson's staining revealed that compared with the $M I$ group, the left ventricular infarction area was lower in the IVA, MET, and IVA + MET groups ( $p<0.05, p<0.05$, and $p<0.01$, respectively), while collagen volume fraction (CVF) also was lower in the other groups (all $p<0.01$ ). The left ventricular infarction area and CVF both were lower in the IVA + MET group, compared to the MET group $(p<0.05$ and $p<0.01$, respectively). The immunohistochemistry and Western blot revealed that $p-C \times 43$ expression was higher in the treatment groups, compared with the MI group (all $p<0.01$ ).

Conclusions: IVA can reduce the incidence of ventricular arrhythmia after $\mathrm{MI}$ in male rats by improving both structural and electrical remodeling, and the combination of IVA and MET is even more effective.

Key words: ivabradine, metoprolol, myocardial infarction, ventricular arrhythmia, connexin 43.

\section{Introduction}

Resting heart rate is a strong independent risk factor in patients with a variety of cardiovascular diseases, including coronary heart disease and

\author{
Corresponding authors: \\ Fei-long Zhang \\ Department \\ of Cardiology \\ Fujian Medical University \\ Union Hospital \\ and Fujian Provincial \\ Institute of Coronary Disease \\ 29 Xinquan Road \\ Gulou District \\ Fuzhou 350001, China \\ Phone: +86 13365910662 \\ Fax: +86059183322156 \\ E-mail: cardizhang@163.com \\ Hang-zhou Wu \\ Medical Insurance Office \\ Fujian Medical \\ University Union Hospital \\ 29 Xinquan Road \\ Gulou District \\ Fuzhou 350001, China \\ Phone: +860591 86218558 \\ Fax: +86059183322156
}


left ventricular dysfunction [1]. At present, the drugs most commonly used to clinically slow the heart rate are $\beta$-blockers. However, for patients with asthma, atrioventricular block, decompensated congestive heart failure, hypotension, or peripheral vascular disease, the application of $\beta$-blockers is restricted to a certain limit. Because ivabradine (IVA) has no negative impact on cardiac muscular strength or the conductive system and is especially suitable for patients with heart failure, it has been used as an alternative to $\beta$-blockers to clinically control the sinus rhythm. The mechanism by which IVA slows spontaneous activity is through inhibiting the pacemaker current (If) by auto-specifically reducing the If conductance, which in turn reduces the pacing frequency of $P$ cells in the sinus node [2]. Meanwhile, IVA has no adverse effects on the atrioventricular conduction system, ventricular systolic and diastolic function, or blood pressure [3].

Heart rate has a substantial influence on the prognosis of ischemic heart disease. Myocardial oxygen consumption increases with heart rate. Furthermore, an elevated heart rate can reduce the oxygen supply of the myocardium and promote both anatomical and electrical remodeling after myocardial infarction (MI) [4]. IVA controls heart rate without affecting the myocardial contractile function, ensuring safety and patient tolerance of the drug. A number of animal studies have revealed that IVA protects cardiac structure and myocardial function from being damaged after acute myocardial infarction (AMI) $[5,6]$. In addition, it can reduce the incidence of ventricular tachycardia (VT)/ventricular fibrillation (VF) after AMI [7]. $\beta$-Blockers, such as metoprolol (MET), have been shown to reconstruct myocardial remodeling, reduce ventricular arrhythmia, and improve prognosis during the repair period of $\mathrm{Ml}$; this has been confirmed by a number of clinical studies and animal trials [8, 9]. Assuming that IVA also has the effect of improving myocardial remodeling and electrical remodeling during the repair period of $\mathrm{MI}$, it is possible that IVA may reduce the occurrence of ventricular arrhythmia.

\section{Material and methods}

\section{Main materials and reagents}

IVA, MET, Cx-43 antibody (ID:3511S), p-Cx43 (Ser368) antibody (ID:3512S), and PVDF membranes were respectively purchased from Servier Pharmaceuticals GmbH (France), AstraZeneca Pharmaceutical Co., Wuhan Sanying Biotechnology Co., Cell Signaling Technology, and Millipore Corporation (USA). Hypersensitive two-step immunohistochemistry detection reagents of rabbit and goat anti-rabbit lgG, and other reagents were purchased from Wuhan Sevia Biotechnology Co.

\section{Experimental animals and grouping}

A total of 60 specific-pathogen-free (SPF) male Sprague-Dawley rats, weighing 230-280 g, were purchased from Shanghai Slack Laboratory Animal Co. (License No.: SCXK (Shanghai) 2012-0002, animal certificate number: 201500054135), and subjected to adaptive feeding for one week. The rats were then randomly divided into five groups according to the random number table, with 12 rats in each group. MI group rats received left anterior descending (LAD) coronary artery ligation and a normal diet; IVA group rats were treated with $10 \mathrm{mg} /(\mathrm{kg} \times$ day) of IVA [10] by gavage after ligation of the LAD coronary artery; MET group rats were treated with $50 \mathrm{mg} /(\mathrm{kg} \times$ day $)$ of $M E T$ [11] by gavage after ligation of the LAD coronary artery; IVA + MET group rats received $10 \mathrm{mg} /(\mathrm{kg} \times$ day) of IVA $+50 \mathrm{mg} /(\mathrm{kg} \times$ day $)$ of MET by gavage after ligation of the LAD coronary artery; and sham group rats received a normal diet and only ligature (without ligation) of the LAD coronary artery. The drug treatment was started $24 \mathrm{~h}$ after ligation of the anterior descending coronary artery. The interventions lasted for 4 weeks. The protocol was approved by ethics committees in Fujian Medical University Union Hospital. During the experiment, the researchers followed the internationally recognized animal welfare guidelines and the Guidelines for the Care and Use of Laboratory Animals published by the National Institutes of Health.

\section{Preparation for the animal model of myocardial infarction}

The rats were intraperitoneally anesthetized with $1 \%$ sodium pentobarbital $(5 \mathrm{ml} / \mathrm{kg})$, and fixed and prepared for skin disinfection. Then, tracheal intubation was performed and cannula was connected to the ventilator. Next, the chest skin was cut at approximately $3 \mathrm{~cm}$ longitudinally alongside the left $3 \mathrm{~mm}$ of the sternum, the pectoralis major and anterior serratus muscle were separated by blunt dissection, the intercostal muscle was cut off along the third intercostal space, and piercing was performed through the pleura. After this, the ribs were opened, a cut was made in the pericardium to reveal the heart, and a 5-0 line was used to suture the proximal LAD coronary artery $(2 \mathrm{~mm}$ below the junction of the pulmonary artery cone and left atrial appendage). Certain observation targets were set as the termini of MI, including the appearance of $>0.2 \mathrm{mV}$ of ST-segment elevation on more than two limb leads, and the myocardium in the left ventricular anterior wall turning from red to white, with its movement restricted. It was assumed that the MI model was successfully established when all the above targets were reached after $10 \mathrm{~min}$ of sur- 
gery. In the process of modeling, the researchers used a blind method to model the group of rats. So did the researchers who did the electrophysiological examination.

\section{Heart rate and blood pressure measurements}

The heart rate and blood pressure of the rats were measured before surgery, and after 4 weeks of $\mathrm{Ml}$ modeling. After starting the non-invasive blood pressure measurement system (BP-2010, Softron), the rats were maintained in a quiet environment for $15 \mathrm{~min}$, and fixed in the device. Then, heart rate and blood pressure were measured once every $5 \mathrm{~min}$, and averaged after three measurements.

\section{Electrophysiological detection [12]}

At 4 weeks after the establishment of the MI model, anesthesia, fixation, connection to the ventilator and exposure of the heart were successively performed. Then, the heart was connected to a multi-conducting physiological recorder (GY6000F, Henan South China Medical) to determine the following parameters in sequence: (1) QRS wave and QT interval, QTc (II lead). (2) Ventricular effective refractory period (VERP). The electrode was vertically placed on the anterior wall of the left ventricle, stimulation was induced from S1S2, and the basic perimeter was set at $130 \mathrm{~ms}$. The S2 stimulation was progressively decreased from $90 \mathrm{~ms}$ to the minimum to attain the VERP. (3) VERP dispersion (VERPd). The VERP of the myocardium in the ventricular anterior wall and side wall were measured, the difference in the value of VERP between any two places was calculated, and the maximum difference was taken as VERPd. (4) Induction rate and fatality rate of VT/VF. The right ventricular anterior wall was stimulated with S1S1 40-ms bursts for $10 \mathrm{~s}$; this was repeated three times, with a 3-minute interval. Then, the induction rate and fatality rate of VT/VF were recorded. The range of VT/VF was $2 \mathrm{~ms}$ when the stimulation output was two times the threshold.

\section{Pathological observation of the left ventricle}

The heart was collected, and the left ventricle was separated along the level of the papillary muscles into two parts: the horizontal part of the papillary muscle, and the apex part. The myocardium of the horizontal part of the papillary muscle was placed in $10 \%$ neutral formalin for $24 \mathrm{~h}$, and dehydrated and paraffin-embedded. Then, the paraffin-embedded sections were consecutively cut into a total of 24 slices, with a thickness of $4 \mu \mathrm{m}$. Under an optical microscope, six slices were stained with hematoxylin and eosin ( $\mathrm{H} \& \mathrm{E})$, ten fields were randomly selected from each slice, and the ratio of the myocardial infarcted area to the total area (percentage) was calculated using the IPP 6.0 image analysis system. Next, six slices were stained with Masson's staining, ten fields were randomly selected from each slice, and the collagen volume fraction (CVF) was calculated using the IPP 6.0 image analysis system. Immunohistochemistry was applied to the remaining 12 slices. After immunohistochemical preparation, 100x microphotographs were taken for each group, and the positive expression of $p-C \times 43$ and $t-C \times 43$ in the left ventricular myocardium of SD rats in each group was detected through the multifunctional true color cell image analysis and management system. The brown cells located at the longitudinal junction and side of the cardiomyocytes were positively expressed. After hematoxylin counterstaining, the cells with blue-black nuclei were the total number of cells, so that the percentage of positive expressing cells could be calculated.

\section{Western blot}

Western blot was performed to detect the expression of $\mathrm{p}-\mathrm{C} \times 43$ and $\mathrm{t}-\mathrm{C} \times 43$ in the left ventricular myocardium. The apex was collected and homogenized for protein extraction and quantification. Then, SDS-PAGE loading buffer was added to $60 \mu \mathrm{g}$ of protein sample, and the sample was heated and centrifuged and applied on 4-12\% gradient precast gel for electrophoresis. A PVDF membrane was used as the solid-phase supporter. Next, the corresponding protein monoclonal antibody was added after transfection and blocking, the bivalent antibody was labeled, and the X-ray tablet was placed on an automatic processor for development and fixation. This was then scanned and analyzed, and the gray value of each band was calculated using the gel image system. The relative protein expression level was presented as the ratio of the gray value corrected by the internal reference GAPDH.

\section{Statistical analysis}

Statistical analysis was performed using SPSS 24.0 statistical software. The measurement data of normal distribution was presented as $x \pm$ standard deviation (SD). A mean-score comparison between groups was performed using variance analysis, which was applied to completely randomized design data. When the results of the variance analysis were found to be statistically significant, an LSD $t$-test was used for further comparison. Non-normally distributed measurement data were presented as $M(\mathrm{Q} 1, \mathrm{Q} 3)$. The non-parametric rank sum test was used for comparison between groups. Count data were presented as the composition ratio. The $\chi^{2}$ test was used for comparison 
between groups. $P<0.05$ was considered statistically significant.

\section{Results}

\section{Variations in blood pressure and heart rate (Table I)}

There was no statistically significant difference in either systolic blood pressure or diastolic blood pressure between any two groups before modeling, or after the 4-week drug intervention (for all, $p>0.05)$. Furthermore, there was no statistically significant difference in heart rate between any two groups before the modeling $(p>0.05)$. After the 4-week drug intervention, there was no significant difference in heart rate between the $\mathrm{MI}$ group and sham group $(p>0.05)$; however, heart rates were significantly slower in the IVA, MET, and IVA + MET groups, compared with the MI group $(p<0.01)$. There was no significant difference in heart rate between the IVA group and MET group ( $p>0.05$ ); however, compared with the MET group, the heart rate was slower in the IVA + MET group $(p<0.01)$.

\section{Electrophysiological detection results (Table II)}

One rat died in the $\mathrm{MI}$ group and another rat died in the MET + IVA group after the MI model was established. After 4 weeks of intervention, and compared with the sham group, QT and QTC interval was prolonged, VERP was shortened, VERPd was higher, and the induction rate and fatality rate of VT/VF were higher in the MI group (all $p<0.01$ ). Compared with the MI group, QT and QTc interval was shorter, VERP was longer and VERPd was lower in the IVA group, MET group, and IVA + MET group (for all, $p<0.01$ ). Compared with the MI group, the induction rate and fatality rate of VT/VF were lower in the IVA group and MET group (for all, $p<0.05$ ); the induction rate

Table I. Heart rate and blood pressure of each group of rats $(x \pm s)$

\begin{tabular}{|c|c|c|c|c|c|c|c|c|}
\hline \multirow[t]{2}{*}{ Variable } & \multicolumn{4}{|c|}{ Before modeling } & \multicolumn{4}{|c|}{ After 4 weeks } \\
\hline & $\begin{array}{c}\text { Number } \\
\text { of rats }\end{array}$ & $\begin{array}{c}\text { Heart rate } \\
\text { [beats/ } \\
\text { min] }\end{array}$ & $\begin{array}{l}\text { Systolic } \\
\text { blood } \\
\text { pressure } \\
\text { [mm Hg] }\end{array}$ & $\begin{array}{l}\text { Diastolic } \\
\text { blood } \\
\text { pressure } \\
\text { [mm Hg] }\end{array}$ & $\begin{array}{c}\text { Number } \\
\text { of rats }\end{array}$ & $\begin{array}{c}\text { Heart rate } \\
\text { [beats/ } \\
\text { min] }\end{array}$ & $\begin{array}{l}\text { Systolic } \\
\text { blood } \\
\text { pressure } \\
\text { [mm Hg] }\end{array}$ & $\begin{array}{c}\text { Diastolic } \\
\text { blood } \\
\text { pressure } \\
{[\mathrm{mm} \mathrm{Hg}]}\end{array}$ \\
\hline MI group & 12 & $404 \pm 9$ & $111 \pm 10$ & $70 \pm 8$ & 11 & $407 \pm 9^{f}$ & $115 \pm 8$ & $69 \pm 6$ \\
\hline IVA group & 12 & $399 \pm 7$ & $114 \pm 9$ & $71 \pm 9$ & 12 & $305 \pm 13^{\text {de }}$ & $116 \pm 9$ & $69 \pm 6$ \\
\hline MET group & 12 & $405 \pm 14$ & $112 \pm 9$ & $69 \pm 9$ & 12 & $314 \pm 10^{\text {de }}$ & $115 \pm 9$ & $72 \pm 7$ \\
\hline IVA + MET group & 12 & $400 \pm 11$ & $113 \pm 9$ & $72 \pm 8$ & 11 & $236 \pm 14^{\text {def }}$ & $115 \pm 8$ & $73 \pm 9$ \\
\hline Sham group & 12 & $406 \pm 10$ & $112 \pm 8$ & $73 \pm 6$ & 12 & $408 \pm 9^{f}$ & $116 \pm 7$ & $72 \pm 7$ \\
\hline$F$ value & & 0.938 & 0.166 & 0.345 & & 494.727 & 0.080 & 0.672 \\
\hline$P$-value & & 0.449 & 0.955 & 0.847 & & 0.000 & 0.988 & 0.614 \\
\hline
\end{tabular}

Compared with the sham group, ${ }^{d} p<0.01$; compared with the MI group, ${ }^{e} p<0.01$; compared with the MET group, ${ }^{f} p<0.01$.

Table II. Electrophysiological test results in each group

\begin{tabular}{|c|c|c|c|c|c|c|c|c|}
\hline Variable & $\begin{array}{l}\text { Number } \\
\text { of rats }\end{array}$ & $\begin{array}{c}\text { QRS } \\
\text { wave } \\
\text { time limit } \\
x \pm s \\
{[\mathrm{~ms}]}\end{array}$ & $\begin{array}{c}\text { QT inter- } \\
\text { phase } \\
x \pm s \\
{[\mathrm{~ms}]}\end{array}$ & $\begin{array}{c}\text { QTc } \\
x \pm s \\
{[\mathrm{~ms}]}\end{array}$ & $\begin{array}{l}\text { VERP } \\
x \pm s \\
{[\mathrm{~ms}]}\end{array}$ & $\begin{array}{c}\text { VERPd } \\
x \pm \mathrm{s}[\mathrm{ms}]\end{array}$ & $\begin{array}{l}\text { VT/VF } \\
\text { inducing } \\
\text { rate } x / n\end{array}$ & $\begin{array}{c}\text { VT/VF } \\
\text { fatality } \\
\text { rate } \\
y / n\end{array}$ \\
\hline MI group & 11 & $21.8 \pm 1.3$ & $78.5 \pm 4.0^{\text {ac }}$ & $204.3 \pm 11.3^{\mathrm{df}}$ & $50.2 \pm 4.7^{\mathrm{df}}$ & $18.6 \pm 2.2^{\mathrm{df}}$ & $10 / 11^{c d}$ & $5 / 11^{\text {ac }}$ \\
\hline IVA group & 12 & $21.9 \pm 1.6$ & $68.6 \pm 4.2^{\mathrm{ab}}$ & $154.7 \pm 10.5^{\mathrm{e}}$ & $60.0 \pm 3.4^{\mathrm{de}}$ & $10.7 \pm 2.7^{\text {de }}$ & $6 / 12^{\mathrm{ab}}$ & $1 / 12^{\mathrm{b}}$ \\
\hline MET group & 12 & $21.8 \pm 1.3$ & $69.1 \pm 5.3^{\mathrm{ab}}$ & $157.9 \pm 12.0^{\mathrm{e}}$ & $60.6 \pm 3.9^{\text {de }}$ & $10.3 \pm 2.7^{\text {de }}$ & $6 / 12^{\mathrm{ab}}$ & $1 / 12^{b}$ \\
\hline IVA + MET group & 11 & $21.6 \pm 1.4$ & $62.7 \pm 3.8^{b c}$ & $124.4 \pm 8.9^{\text {def }}$ & $64.1 \pm 2.9^{\text {ce }}$ & $7.4 \pm 2.0^{\text {ef }}$ & $1 / 11^{\text {ce }}$ & $1 / 11^{b}$ \\
\hline Sham group & 12 & $21.7 \pm 1.4$ & $60.7 \pm 4.8^{\mathrm{bc}}$ & $158.1 \pm 12.2^{\mathrm{e}}$ & $66.2 \pm 3.7^{\text {ef }}$ & $5.8 \pm 2.3^{\mathrm{ef}}$ & $1 / 12^{\text {ce }}$ & $1 / 12^{\mathrm{b}}$ \\
\hline$F$ value & & 0.129 & 27.083 & 73.020 & 30.380 & 46.524 & & \\
\hline$P$-value & & 0.971 & 0.000 & 0.000 & 0.000 & 0.000 & & \\
\hline
\end{tabular}

Compared with the sham group, ${ }^{a} p<0.05$; compared with the MI group, ${ }^{b} p<0.05$; compared with the MET group, ${ }^{c} p<0.05$; compared with the sham group, ${ }^{d} p<0.01$; compared with the MI group, ${ }^{e} p<0.01$; compared with the MET group, ${ }^{f} p<0.01$. 
and fatality rate of VT/VF also were lower in the IVA + MET group $(p<0.01$ and $p<0.05$, respectively). No statistically significant difference was observed between the MET group and IVA group in QT and QTc interval, VERP, VERPd, or induction rate and fatality rate of VT/VF (all $p>0.05$ ). Compared with the MET group, the QT and QTc interval was shorter $(p<0.01)$, VERP was longer $(p<0.05)$, and VERPd and the induction rate of VT/VF were lower (both $p<0.05$ ) in the IVA + MET group, but there was no statistically significant difference in the fatality rate of VT/VF $(p>0.05)$.

\section{H\&E staining results (Figures 1 and 2)}

In the sham group, the left ventricular cardiomyocytes were neatly arranged, while in the $\mathrm{MI}$ group, large infarctions had formed, including the fragmentation of cellular nucleuses and increased infiltration of chronic inflammatory cells. The left ventricular infarction areas $(x \pm S D, \%)$ in the MI, IVA, MET, IVA + MET, and sham groups were 28.3 $\pm 7.4 \%, 10.5 \pm 2.5 \%, 10.8 \pm 2.5 \%, 5.9 \pm 1.1 \%$, and 0.0 $\pm 0.0 \%$, respectively. The left ventricular infarction area was larger in the MI group than in the sham group $(p<0.01)$; however, it was smaller in each of the mg IVA, MET, and IVA + MET groups than in the MI group ( $p<0.05, p<0.05$, and $p<0.01$, respectively). While no statistically significant difference was observed in left ventricular infarction area between the MET group and IVA group $(p>0.05)$, in

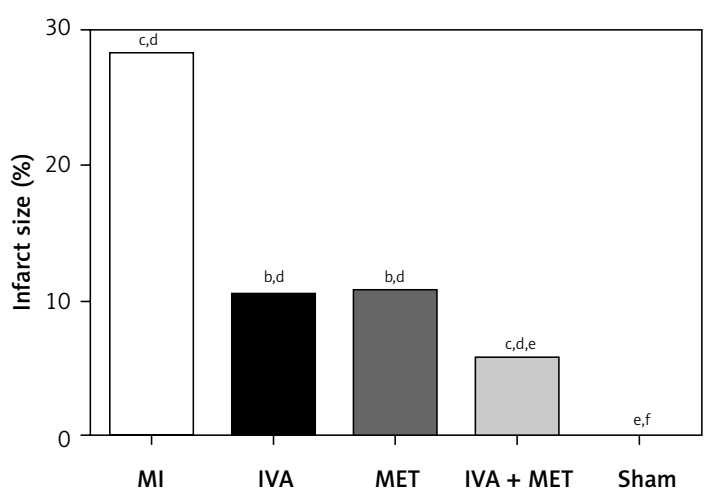

Figure 1. Area ratio of each group of myocardial infarction rats. Compared with the sham group, ${ }^{\mathrm{a}} p<0.05$; compared with the Ml group, ${ }^{\mathrm{b}} p<0.05$; compared with the MET group, ${ }^{c} p<0.05$; compared with the sham group, ${ }^{d} p<0.01$; compared with the MI group, ${ }^{e} p<0.01$; compared with the MET group, ${ }^{\mathrm{f}} p<0.01$

the IVA + MET group the left ventricular infarction area was smaller than in the MET group $(p<0.05)$.

\section{Masson's staining results (Figures 3 and 4)}

The ventricular myocardial CVF $(x \pm S D, \%)$ in the MI, IVA, MET, IVA + MET, and sham groups was $21.4 \pm 3.2 \%, 12.5 \pm 2.5 \%, 13.0 \pm 3.1 \%, 6.5 \pm 1.4 \%$ and $1.3 \pm 1.4 \%$, respectively. The left ventricular CVF was larger in the $\mathrm{MI}$ group than in the sham group ( $p<0.01)$, and smaller in the IVA, MET, and IVA + MET groups than in the MI group $(p<0.01)$.

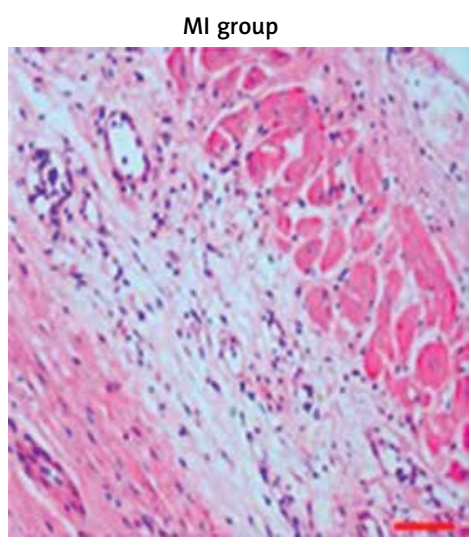

IVA + MET group

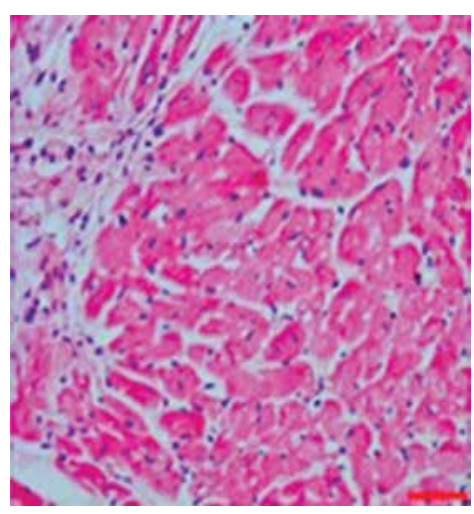

IVA group

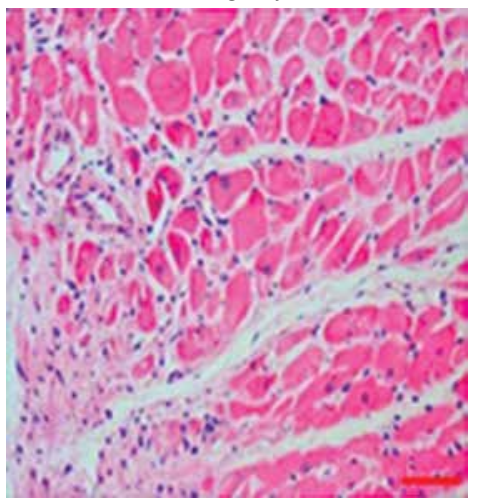

Sham group

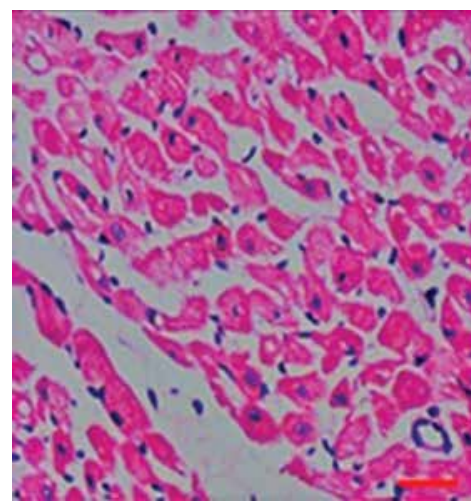

MET group

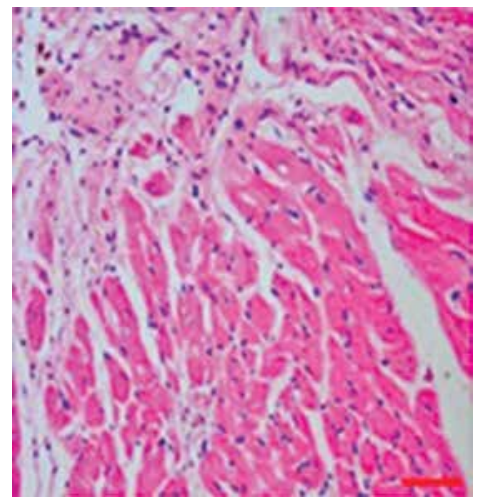

Figure 2. HE staining ventricular muscle tissue (200x, calibrated to $100 \mu \mathrm{m}$ ) 


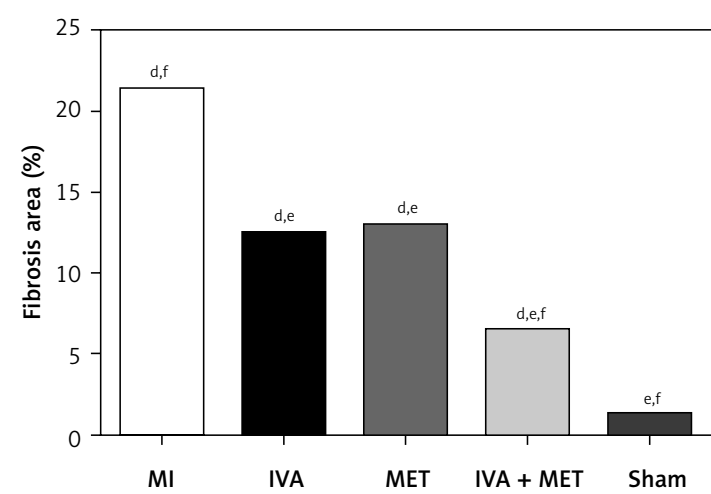

Figure 3. Fibrosis area (\%) of the left ventricular anterior wall of the myocardium in each group. Compared with the sham group, ${ }^{\mathrm{d}} p<0.01$; compared with the MI group, ${ }^{\mathrm{e}} p<0.01$; compared with the MET group, ${ }^{f} p<0.01$

While no significant difference was observed in the left ventricular CVF between the IVA group and MET group $(p>0.05)$, the left ventricular CVF was smaller in the IVA + MET group than in the MET group $(p<0.01)$.

\section{Distribution and expression of $\mathrm{p}-\mathrm{C} \times 43$} and $\mathrm{t}-\mathrm{C} \times 43$ in the left ventricle myocardium (immunohistochemistry: Table III and Figure 5)

Compared with the sham group, $\mathrm{p}-\mathrm{C} \times 43$ protein expression was lower in the MI group $(p<0.01)$; it was greater in the IVA, MET, and IVA + MET groups than in the MI group $(p<0.01)$. While there was no statistically significant difference in $\mathrm{p}-\mathrm{C} \times 43$ protein expression between the IVA group and MET group $(p>0.05), p-C \times 43$ protein expression was greater in the IVA + MET group than in the MET group $(p<0.01)$. However, there was no statistically significant difference in left ventricular anterior wall t-Cx43 expression between any two groups $(p>0.05)$. In the sham group, both the $\mathrm{p}-\mathrm{C} \times 43$ and $\mathrm{t}-\mathrm{C} \times 43$ protein were almost uniformly distributed in the longitudinal junction of the myocardium. In the MI group, the majority of the $\mathrm{p}-\mathrm{C} \times 43$ and $\mathrm{t}-\mathrm{C} \times 43$ proteins were distributed in the lateral-lateral junction of myocardial cells, while few were distributed in the longitudinal junction of the myocardium. The distribution of $\mathrm{p}-\mathrm{C} \times 43$ and $\mathrm{t}-\mathrm{C} \times 43$ proteins in the IVA, MET, and IVA + MET groups was somewhere between the distributions in the sham and MI groups.

\section{Protein expression of $\mathrm{p}-\mathrm{C} \times 43$ and $\mathrm{t}-\mathrm{C} \times 43$ in the left ventricle myocardium (Western blot: Table IV and Figure 6)}

Compared with the sham group, $\mathrm{p}-\mathrm{C} \times 43$ protein expression was lower in the MI group ( $p<0.01)$; it was greater in the IVA, MET, and IVA + MET groups than in the MI group $(p<0.01)$. While there was no statistically significant difference in $\mathrm{p}-\mathrm{C} \times 43$

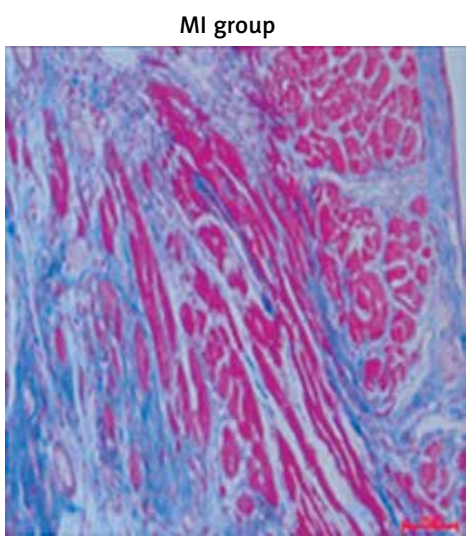

IVA + MET group

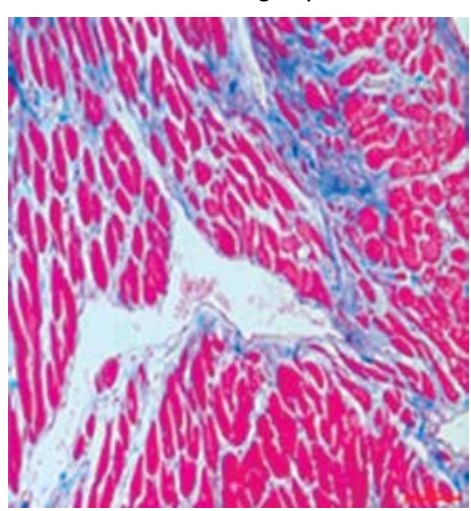

IVA group

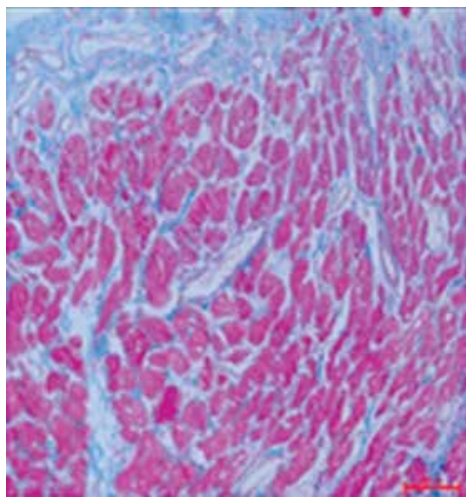

Sham group

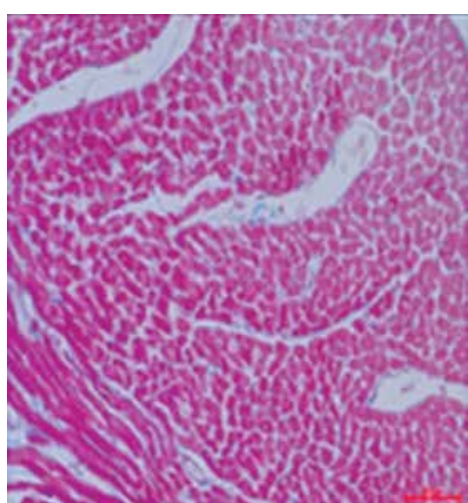

MET group

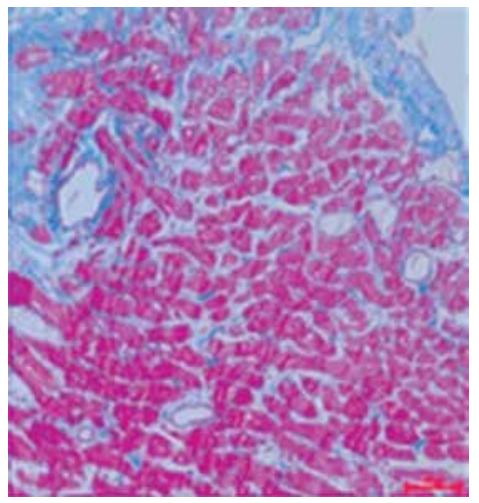

Figure 4. Masson staining ventricular muscle tissue (100 fold, calibrated to $200 \mu \mathrm{m}$ ) 
Table III. $\mathrm{p}-\mathrm{C} x 43, \mathrm{t}-\mathrm{C} \times 43$ positive cell percentage and score $(x \pm \mathrm{s})$

\begin{tabular}{|lccccc|}
\hline Variable & Number of rats & \multicolumn{2}{c}{$\mathrm{p}$-Cx43 } & \multicolumn{2}{c|}{$\mathrm{t}$-Cx43 } \\
\cline { 3 - 6 } & & A (\%) & B & A (\%) & B \\
\hline MI group & 11 & $31 \pm 4^{\text {df }}$ & 2 & $88 \pm 3$ & 2 \\
\hline IVA group & 12 & $65 \pm 5^{\text {de }}$ & 3 & $92 \pm 2$ & 2 \\
\hline MET group & 12 & $63 \pm 7^{\text {de }}$ & 3 & $89 \pm 6$ & 2 \\
\hline IVA + MET group & 11 & $76 \pm 4^{\text {def }}$ & 3 & $90 \pm 3$ & 2 \\
\hline Sham group & 12 & $91 \pm 5^{\text {ef }}$ & 3 & $87 \pm 6$ & 0.837 \\
\hline F value & & 83.719 & & 0.523 & 2 \\
\hline P-value & & 0.000 & & 3 \\
\hline
\end{tabular}

$A$ is the percentage of positive cells. $B$ is color intensity grade of positive cells; 0 (negative), 1 (weak positive), 2 (positive), 3 (strong positive). Compared with the sham group, ${ }^{d} p<0.01$; compared with the MI group, ${ }^{e} p<0.01$; compared with the MET group, ${ }^{f} p<0.01$.

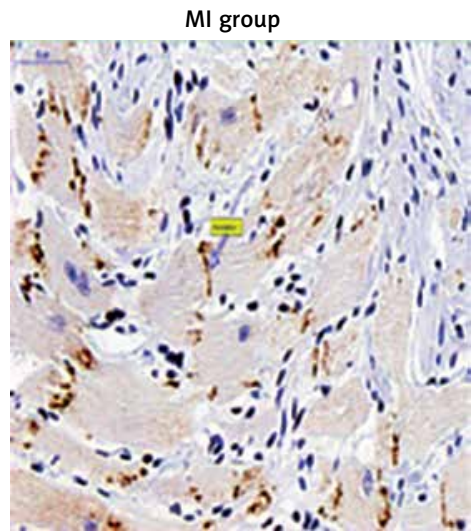

IVA + MET group

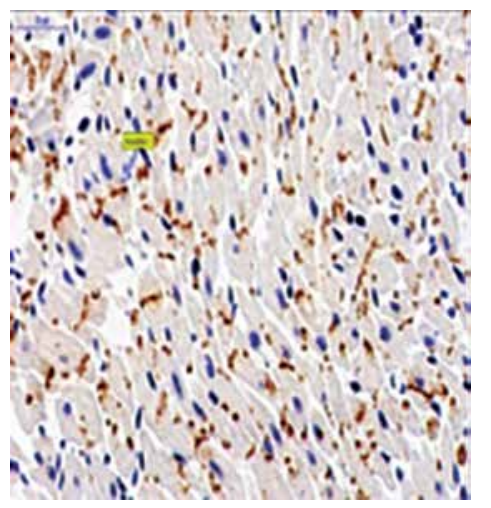

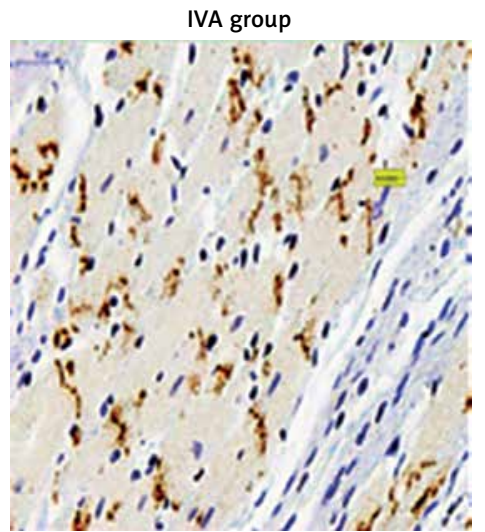

Sham group

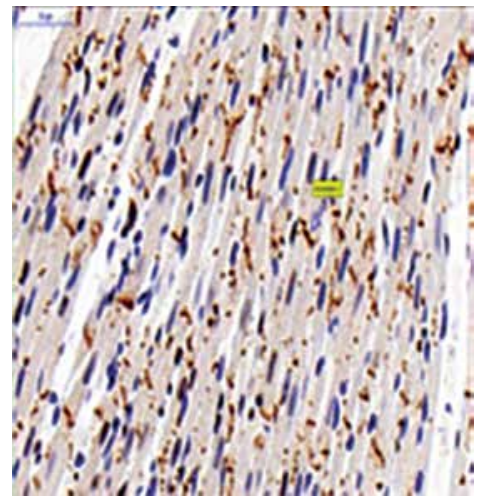

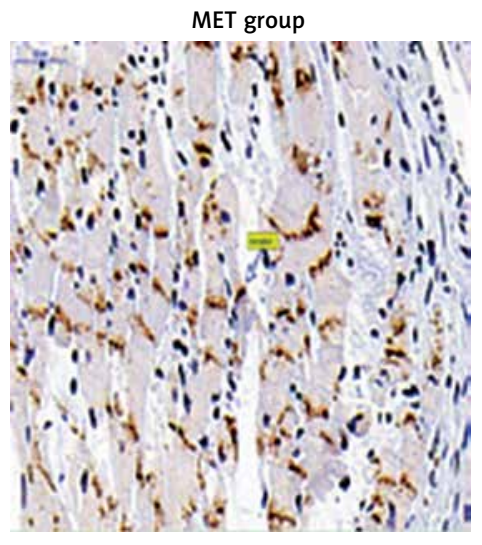

Figure 5. Immunohistochemical staining of $\mathrm{p}-\mathrm{Cx} 43(400 \times$, scaled at $50 \mu \mathrm{m})$. The myocardial cell lateral and longitudinal junctions are brown, and are positively expressed, with the wood grain re-dyeing nuclei in dark blue protein expression between the IVA group and the MET group $(p>0.05), p-C \times 43$ protein expression was greater in the IVA + MET group than in the MET group $(p<0.05)$. However, there was no statistically significant difference in left ventricular anterior wall $\mathrm{t}-\mathrm{C} \times 43$ expression between any two groups $(p>0.05)$.

\section{Discussion}

Myocardial infarction is myocardial necrosis caused by the interruption of coronary blood flow $[13,14]$. Partial cardiomyocyte necrosis oc- curs due to severe and persistent ischemia [15]. Myocardial infarction results in high morbidity and mortality worldwide [16]. Only male rats were used in this study in order to control for the effects of gender. Although the current guidelines recommend an equal approach, a previous study found that in patients with non-ST-segment elevation myocardial infarction (NSTEMI), after age adjustment, mortality rates were similar in both genders [17]. Ventricular arrhythmia after myocardial infarction is one of the main causes of death in patients with myocardial infarction, especially 
Table IV. $\mathrm{P}-\mathrm{C} \times 43$ and $\mathrm{t}-\mathrm{C} \times 43$ protein expression in each group $(x \pm s)$

\begin{tabular}{|c|c|c|c|}
\hline Variable & $\begin{array}{l}\text { Number } \\
\text { of rats }\end{array}$ & $p-C \times 43$ & $t-C \times 43$ \\
\hline MI group & 11 & $0.17 \pm 0.02^{\mathrm{df}}$ & $0.70 \pm 0.03$ \\
\hline IVA group & 12 & $0.28 \pm 0.01^{\mathrm{de}}$ & $0.72 \pm 0.04$ \\
\hline MET group & 12 & $0.28 \pm 0.02^{\mathrm{de}}$ & $0.71 \pm 0.02$ \\
\hline $\begin{array}{l}\text { IVA + MET } \\
\text { group }\end{array}$ & 11 & $0.34 \pm 0.04^{\text {cde }}$ & $0.70 \pm 0.03$ \\
\hline Sham group & 12 & $0.44 \pm 0.03^{\mathrm{ef}}$ & $0.71 \pm 0.03$ \\
\hline$F$ value & & 306.158 & 0.897 \\
\hline$P$-value & & 0.000 & 0.473 \\
\hline
\end{tabular}

Compared with the MET group, ${ }^{c} p<0.05$; compared with the sham group, ${ }^{d} p<0.01$; compared with the MI group, ${ }^{e} p<0.01$; compared with the MET group, $f p<0.01$.

in the pre-hospital stage, which poses a persistent problem [18]. Among the drugs for secondary prevention of coronary heart disease, angiotensin-converting enzyme inhibitors and statins have been confirmed to improve ventricular remodeling after MI, but have limited effects in the prevention and treatment of secondary ventricular arrhythmias. Furthermore, various studies have proven that $\beta$-blockers are beneficial in reducing the occurrence of ventricular arrhythmias after MI [19, 20], and improving the clinical prognosis during the repair period of MI. At present, based on the guidelines, $\beta$-blockers (such as metoprolol succinate) are recommended for the prevention and treatment of ventricular arrhythmias after $\mathrm{MI}$ [21]. The mechanism of $\beta$-blockers is to inhibit excessive sympathetic nervous system activity [19], reduce myocardial oxygen consumption, protect the myocardium [22], prevent triggered activity and re-entry induced by effective refractory period shortening, inhibit the downregulation of $\mathrm{p}-\mathrm{C} \times 43$ in the $\mathrm{MI}$ area, improve the spatial redistribution of $p-C \times 43$, and increase the threshold of ventricular fibrillation [11]. Cx43 is an important part of the gap junction channel, and $\mathrm{p}-\mathrm{C} \times 43$ participates in the formation of the functional gap junction channel. Cx43 is the main protein responsible for ventricular electrical conduction. Changes in its protein expression level and spatial distribution, and in $\mathrm{p}-\mathrm{C} \times 43$ state, can all lead to electrical remodeling, which is related to the occurrence of arrhythmia. The conformation of $\mathrm{C} \times 43$ in ventricular myocytes changes rapidly during myocardial infarction. Its expression is down-regulated and mainly distributed laterally, leading to the inactivation of gap junctions, blocking the transverse conduction velocity more than longitudinally and causing uncoupling of electrical impulses between cells, delay or blockage of action potential conduction, and formation of re-entrant loops, which

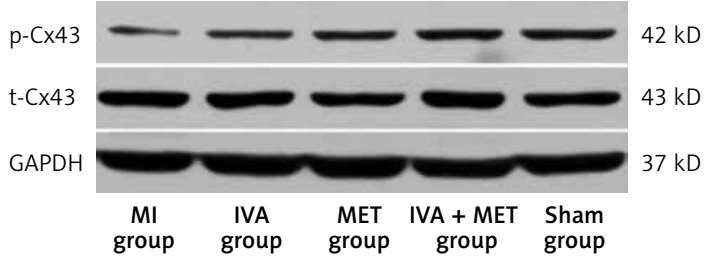

Figure 6. Groups of the left ventricular myocardium $\mathrm{p}-\mathrm{C} \times 43$ and $\mathrm{t}-\mathrm{C} \times 43$ protein expression

plays an important role in the occurrence of ventricular arrhythmia. Previous studies have shown that $\mathrm{p}-\mathrm{C} \times 43$ was down-regulated after myocardial infarction, and that the spatial distribution was abnormal, resulting in a decrease in the end-toend distribution of cardiomyocytes and an increase in lateral distribution. The up-regulated expression of $p-C \times 43$, and the increase of endto-end distribution in cardiomyocytes, can reduce the occurrence of arrhythmia after myocardial infarction [23].

To date, the exact mechanism of ventricular arrhythmia after $\mathrm{MI}$ has not been fully elucidated. It is clearer that the structural and electrical remodeling of myocardial cells after MI induce instability in electrical and mechanical activity. This feature contributes to the occurrence of related ventricular arrhythmias after MI [24].

Neuro-humoral and mechanical changes after $\mathrm{MI}$ promote the transformation of mesenchymal cells into myofibroblasts and accelerate myocardial fibrosis [25]. In addition, the ischemic adaptive changes after $\mathrm{MI}$ induce myocardial cell hypertrophy, which causes the diffusion and conduction velocity of electrical impulses to decrease, and the ectopic pacing to perform as ventricular premature contraction [26]. Myocardial fibrosis and hypertrophy cause cardiomyocyte fibers to be disordered, which in turn alters cell anisotropy and cell-to-cell coupling [27], and this may lead to desynchronization between ventricular electrical conduction and mechanical activities. Studies have reported that IVA can improve myocardial remodeling, increase capillary density, enhance left ventricular systolic function, and retard myocardial remodeling after MI [7]. A 2017 clinical study showed that patients with acute ST-segment elevation myocardial in farction had a good tolerance to IVA, and IVA was as effective as metoprolol, which can reduce heart rate and reduce the risk of atrioventricular block [28]. In addition, there are several studies on the safety and efficacy of IVA in patients with AMI. However, since IVA has been mostly used in patients with acute heart failure, there were few data on the clinical or preclinical long-term survival time of IVA after MI. In the present study, after the $\mathrm{MI}$ model was established for 4 weeks, H\&E and Masson's staining revealed that in the sham group, the cardiomyocytes were neatly arranged, 
no infarctions were found, and no collagen fibers were deposited in interstitial cells. In the MI group, large infarctions had formed, and collagen fibers were obviously deposited in interstitial cells. Compared with the MI group, both infarction area and collagen fibrosis deposition were significantly reduced after drug intervention. Furthermore, while the infarction area and collagen fibrosis deposition were similar in the IVA group and the MET group, they were lower in the IVA + MET group than in the groups treated with either IVA or MET alone. Compared with the MI group, the heart rate in other groups after drug intervention was evidently slower. The effect on slowing the heart rate was similar in the MET and IVA groups, and, again, the combination of IVA and MET was found to be more effective. Pathohistological results further revealed that there was no significant difference between IVA and MET in improving myocardial structure remodeling and reducing the size of the infarction area after MI. The combination of IVA and MET was more effective than the use of MET alone, which is probably associated with the longterm slowing of the heart rate.

Researchers have recently studied the occurrence of ventricular arrhythmias in electrocardiograms after $24 \mathrm{~h}$ of $\mathrm{AMI}$ in rats, and found that the incidence of VT/VF after AMI was remarkably reduced in the IVA group [8]. Another important mechanism of ventricular arrhythmia after $\mathrm{MI}$ is electrical remodeling. Cx43 is the major gap junction protein responsible for ventricular electrical conduction. Cx43 protein expression levels, spatial distribution and $\mathrm{p}-\mathrm{C} \times 43$ status changes can lead to electrical remodeling, and are associated with the occurrence of arrhythmia. When MI occurs, the conformation of $\mathrm{Cx} 43$ in ventricular cardiomyocytes rapidly changes, and this expression is downregulated. In addition, the structural remodeling of $\mathrm{C} \times 43$ is mainly characterized by cell surface lateralization along cell membranes. The above characteristics lead to the inactivation of gap connections, resulting in slow conduction. Longitudinal conduction was slowed to some extent, while transverse conduction was considerably slowed, causing a reduction in cell-to-cell coupling, and action potential conduction delay or block. The formation of a re-entrant circuit plays an important role in the occurrence of ventricular arrhythmia [29]. Pathologically, the Cx43 gap junction remodeling manifests as downregulated expression, protein dephosphorylation, and the lateral distribution of cardiomyocytes. The present study suggested that reversing the $\mathrm{Cx} 43$ remodeling can restore normal myocardial function and electrical stability. The immunohistochemical results in the present study confirm that $\mathrm{t}-\mathrm{C} \times 43$ and $\mathrm{p}-\mathrm{C} \times 43$ protein in the left ventricular anterior wall in the sham group were mostly distributed in the longitudinal connection of the myocardium. In the Ml group, the distribution of $\mathrm{t}-\mathrm{C} \times 43$ and $\mathrm{p}-\mathrm{C} \times 43$ protein was disorganized, and the cardiomyocytes were mainly distributed in the lateral side. Furthermore, fewer cells were distributed in the longitudinal junction of the myocardium. The immunohistochemistry and Western blot results both confirm that the protein expression of $p-C \times 43$ in the left ventricular anterior wall significantly decreased in the $\mathrm{Ml}$ group; that is, the protein expression of $\mathrm{p}-\mathrm{C} \times 43$ in the left ventricular anterior wall increased after drug intervention. While there was no significant difference between the sole use of IVA and the sole use of MET, a combination of IVA and MET increased the $\mathrm{p}-\mathrm{C} \times 43$ protein level. In addition, the induction rate and fatality rate of VT/VF significantly increased in the $\mathrm{MI}$ group, and both decreased after drug intervention. Again, while there was no significant difference between the sole use of IVA and the sole use of MET, the combination of IVA and $M E T$ reduced the induction rate and fatality rate of VT/VF. In the present study, it was found that IVA mitigated the downregulation of myocardial $\mathrm{p}-\mathrm{Cx} 43$ protein levels after MI, which can effectively reverse the imbalance of ischemia-mediated spatial distribution of $\mathrm{C} \times 43$ and reduce the occurrence of ventricular arrhythmia after $\mathrm{MI}$ in male rats.

The susceptibility to ventricular arrhythmia can be judged through the following indicators: QT interval, VERP and VERPd, and ventricular arrhythmia induction and fatality rates. (1) QT interval represents the depolarization and repolarization time of a ventricle, and its prolongation is directly correlated with the occurrence of ventricular arrhythmia. The QTc interval is the corrected QT interval according to the heart rate. In the MI group, the QT interval was significantly prolonged. After drug intervention, the QT interval was shortened, to the same extent when either IVA or MET was used alone, and to the most extreme extent when a combination of IVA and MET was used. The results for the QTc interval show the same pattern. (2) VERP reflects the excitability of ventricles. The shorter the VERP is, the more likely is the occurrence of re-entry. In the $\mathrm{MI}$ group, the VERP of the left ventricle was significantly shortened, and the risk of ventricular arrhythmia increased. The VERP was prolonged after drug intervention, with no significant difference observed between the IVA group and MET group, but a longer VERP was observed after the use of a combination of IVA and MET. (3) VERPd is a reflection of the heterogeneity of cardiomyocytes in the ventricles, mitral valve, and tricuspid valve. The greater the dispersion, the more obvious the electrophysiological heterogeneity of the ventricular cardiomyocytes. Ventricular arrhythmia can easily form and persist 
in this situation. In the MI group, the VERPd was significant. After drug intervention, the VERPd decreased; it was shortest after a combination of IVA and MET was used, which corresponded to the prior inference of the investigators. The present study indicates that after MI, both IVA and MET can not only reduce myocardial necrosis and fibrosis, but also alleviate the downregulation of $\mathrm{p}-\mathrm{C} \times 43$ and improve its redistribution. Furthermore, the occurrence of VT/VF can be lessened by reducing the susceptibility to ventricular arrhythmia after MI. A combination of IVA and MET is more effective than the use of either one on its own. The outcomes of the present study provide a theoretical basis for the application of IVA in the prevention and treatment of ventricular arrhythmia after MI.

First, no macroscopic or echocardiographic assessment of ventricular remodeling was performed in male rats in this study, which can be improved by future studies. Second, due to the limited methodological possibilities, the quality of the connexin-43 positioning image (Figure 5) cannot be improved.

\section{Acknowledgments}

Li-ping Jiang and Jing-jing Lin contributed equally to this study and should be regarded as co-first authors.

Fund programs: Medical Innovation Project of Fujian Province (2016-CX-17).

\section{Conflict of interest}

There are no interest of conflict associated with this article among all authors.

\section{References}

1. Fox K, Ford I, Steg PG, Tendera M, Robertson M, Ferrari R; BEAUTIFUL investigators. Heart rate as a prognostic risk factor in patients with coronary artery disease and left-ventricular systolic dysfunction (BEAUTIFUL): a subgroup analysis of a randMlsed controlled trial. Lancet 2008; 372: 817-21.

2. DiFrancesco D. Funny channels in the control of cardiac rhythm and mode of action of selective blockers. Pharmacol Res 2006; 53: 399-406.

3. Savelieva I, Camm AJ. If inhibition with ivabradine: electrophysiological effects and safety. Drug Saf 2008; 31: 95-107.

4. Fontes MS, van Veen TA, de Bakker JM, van Rijen HV. Functional consequences of abnormal $\mathrm{C} \times 43$ expression in the heart. Biochim Biophys Acta 2012; 1818: 2020-9.

5. Suffredini S, Stillitano F, Comini L, et al. Long-term treatment with ivabradine in post-myocardial infarcted rats counteracts f-channel overexpression. Br J Pharmacol 2012; 165: 1457-66.

6. Ng FS, Shadi IT, Peters NS, Lyon AR. Selective heart rate reduction with ivabradine slows ischaemia-induced electrophysiological changes and reduces ischaemia-reperfusion-induced ventricular arrhythmias. J Mol Cell Cardiol 2013; 59: 67-75.
7. Mackiewicz U, Gerges JY, Chu S, et al. Ivabradine protects against ventricular arrhythmias in acute myocardial infarction in the rat. J Cell Physiol 2014; 229: 813-23.

8. Wu XL. Amiodarone combined metoprolol for arrhythmia in coronary heart disease clinical curative effect observation. Capital Med 2018; 25: 30-1.

9. Zhou M, Lu Q, Jiang JQ, et al. Succinic acid metoprolol early intervention on rabbit heart tissue of old myocardial infarction effect CX43 and P-CX43 level. Chin J Cardiovasc Dis 2017; 45: 294-8.

10. Maczewski M, Mackiewicz U. Effect of metoprolol and ivabradine on left ventricular remodelling and $\mathrm{Ca} 2+$ handling in the post-infarction rat heart. Cardiovasc Res 2008; 79: 42-51.

11. Babick A, Elimban V, Zieroth S, Dhalla NS. Reversal of cardiac dysfunction and subcellular alterations by metoprolol in heart failure due to myocardial infarction. J Cell Physiol 2013; 228: 2063-70.

12. Zhang ZN, He Y, Zeng ZY, et al. Effects of sympathetic nerve on quickly right atrial pacing induce paroxysmal atrial fibrillation in dogs. Chin Elderly Cardio-cerebrovasc Dis 2014; 16: 189-91.

13. Kocayigit I, Yaylaci S, Osken A, et al. Comparison of effects of thrombolytic therapy and primary percutaneous coronary intervention in elderly patients with acute ST-segment elevation myocardial infarction on in-hospital, six-month, and one-year mortality. Arch Med Sci Atheroscler Dis 2019; 4: e82-e88.

14. Gorący J, Kaczmarczyk M, Ciechanowicz A, et al. E-selectin gene haplotypes are associated with the risk of myocardial infarction. Arch Med Sci 2019; 15: 1223-31.

15. Huang YF, Kang Li. Effects of F cinepolis joint metoprolol on cardiac function in patients with coronary heart disease (CHD) heart failure. World Latest Medicine Information 2015; 15: 129, 131.

16. Cinar H, Avci A, Gulen M, et al. Does stress hyperglycemia affect mortality? Acute myocardial infarction - case control study. Arch Med Sci Atheroscler Dis 2019; 4: e201-7.

17. Piątek $Ł$, Wilczek K, Kurzawski J, et al. Gender-related disparities in the treatment and outcomes in patients with non-ST-segment elevation myocardial infarction: results from the Polish Registry of Acute Coronary Syndromes (PL-ACS) in the years 2012-2014. Arch Med Sci 2020; 16: 781-8.

18. Huang Y, Khanvilkar KH, Moore AD, Hilliard-Lott M. Effects of manufacturing process variables on in vitro dissolution characteristics of extended-release tablets formulated with hydroxypropyl methylcellulose. Drug Dev Ind Pharm 2003; 29: 79-88.

19. Huang YT. Clinical research of Succinic acid metoprolol for the treatment of ventricular arrhythmia after myocardial infarction. J Clin Med Practice 2017; 21: 159-60.

20. Chen ZM, Pan HC, Chen YP, et al.; COMMIT (ClOpidogrel and Metoprolol in Myocardial Infarction Trial) collaborative group. Early intravenous then oral metoprolol in 45,852 patients with acute myocardial infarction: randMIsed placebo-controlled trial. Lancet 2005; 366 : 1622-32.

21. Sheng WF, Zhang Q, Zhang RY. The parsing for diagnosis and treatment of acute ST segment elevation myocardial infarction guidelines in 2015. Int J Cardiovasc Dis 2015; 42: 217-9.

22. Omerovic E, Bollano E, Soussi B, Waagstein F. Selective beta1-blockade attenuates post-infarct remodelling without improvement in myocardial energy metabolism 
and function in rats with heart failure. Eur J Heart Fail 2003; 5: 725-32.

23. Lu Q, Li W, Li Z, et al. Effect of autophagy on cardiomyocyte membrane $\mathrm{C} x 43$ acute remodeling in rats with ischemia-reperfusion. Int J Clin Exp Pathol 2019; 12: 2639-45.

24. Go AS, Mozaffarian D, Roger VL, et al.; American Heart Association Statistics Committee and Stroke Statistics Subcommittee. Heart disease and stroke statistics--2013 update: a report from the American Heart Association. Circulation 2013; 127: e6-245.

25. Davis J, Molkentin JD. Myofibroblasts: trust your heart and let fate decide. J Mol Cell Cardiol 2014; 70: 9-18.

26. Dhein S, Seidel T, Salameh A, et al. Remodeling of cardiac passive electrical properties and susceptibility to ventricular and atrial arrhythmias. Front Physiol 2014; 5: 424.

27. Punske BB, Taccardi B, Steadman B, et al. Effect of fiber orientation on propagation: electrical mapping of genetically altered mouse hearts. J Electrocardiol 2005; 38 (4 Suppl): 40-4.

28. Priti K, Ranwa BL, Gokhroo RK, et al. Ivabradine vs metoprolol in patients with acute inferior wall myocardial infarction -"Expanding arena for ivabradine". Cardiovasc Ther 2017; 35: e12266.

29. Cabo C, Boyden PA. Heterogeneous gap junction remodeling in reentrant circuits in the epicardial border zone of the healing canine infarct. Cardiovasc Res 2006; 72: 241-9. 
Li-ping Jiang, Jing-jing Lin, Ting-pei Zhuang, Wei-wei Wang, Hang-zhou Wu, Fei-long Zhang 
Li-ping Jiang, Jing-jing Lin, Ting-pei Zhuang, Wei-wei Wang, Hang-zhou Wu, Fei-long Zhang 